\title{
COOPERATIVE SPECTRUM SENSING SCHEME USING FUZZY LOGIC TECHNIQUE
}

\author{
Rohit Kantikar and R.G. Yelalwar \\ Department of Electronics and Communications Engineering, Pune Institute of Computer Technology, India
}

\begin{abstract}
In communication system spectrum has a crucial role and wireless technologies are increasing rapidly it is required to make efficient use of spectrum to satisfy the spectrum scarcity problem. Using spectrum efficiently can be done by cognitive radio because of its ability to sense surrounding environment. Cognitive radio sense unoccupied spectrum by detecting the primary users' presence or absence in the spectrum. Using more than one cognitive radio in the detection process will increase the efficiency of spectrum usage and prevent interference between signals. Using machine learning techniques for the implementation of intelligent cognitive radio increase efficiency and detection performance and detect signal at low SNR condition. Fuzzy logic machine learning technique is implemented which is based on fuzzy membership functions and fusion centre. Energy detection is used to classify signal and noise at each cognitive radio after that each cognitive radio output information convert into membership function and apply fuzzy rules such as algebraic sum, the algebraic product give a final decision about the signal presence or absence. Simulation results show that the proposed system gives much better results compared to the conventional energy detection system and improves the performance of the system.
\end{abstract}

\section{Keywords:}

Cognitive Radio, Energy Detection, Cooperative Spectrum Sensing, Fuzzy Logic, Membership Functions

\section{INTRODUCTION}

Nowadays with the technology and the science developing the spectrum was nearly fully occupied. In the communications industry, the number of users increasing rapidly with the use of mobile phones, internet access, data transfer which mainly require high-speed data transmission. So, in that case, Cognitive radios especially aimed at improving the utilization of the electromagnetic spectrum.

The problem of spectrum scarcity reduction is the main purpose of cooperative spectrum sensing using a fuzzy logic technique where unused frequency range in the spectrum is detected using fuzzy logic techniques. The objective is to implement Cooperative spectrum sensing in Cognitive Radio Network based on Machine learning technique particularly fuzzy logic technique and compare the performance of machine learning technique with normal cooperative sensing technique.

Cognitive Radio is a wireless device which can act as the transceiver and identify the spectrum availability status and adapt to the surrounding environment. Cognitive radio avoids channels that are being used, hence ensuring optimum use of the available spectrum while minimizing interference to the other users [1]. It is hybrid technology which adapts to the frequency of communication, encrypts and decrypt the signal sense nearby functioning wireless devices.
The main function of Cognitive radio is a spectrum sensing in which it helps to detect the unused spectrum by the primary user so that the unused spectrum can utilize by other unlicensed users to make efficient utilization of spectrum [2]. So, it is important to detect the transmission of primary user in the spectrum the processing of searching unused spectrum is one of the primary processes of cognitive radio So sensing environment by discovering vacant holes in the spectrum and utilizing that band without damaging primary user and when the primary user starts their transmission immediately vacant the frequency spectrum [3].

Only on one cognitive radio spectrum sensing cannot depends because if any barrier occurs between the primary user and the secondary user then cognitive radio gives incorrect decision and interference will happen between primary user and secondary user so to improve sensing and output accuracy also problems like interference, shadowing fading and hidden node problem group of cognitive radios used so depend on each cognitive radio decision the presence or absence of primary user transmission in spectrum is detected that group of cognitive radio will cooperatively involve in final decision of Primary user presence so-called cooperative Spectrum sensing techniques [4].

In a Centralized Cooperative spectrum sensing network consist of fusion centre which mainly collects data or decision from each cognitive radio of cooperative spectrum sensing and depending on decisions fusion centre applies rules and gives accurate, consistent decision provided by each cognitive radio individual. In cooperative spectrum sensing consist of two segments first one is the detection of primary user presence by each individual cognitive radio defined as local sensing and second stage involve sending data or decision of each cognitive radio to fusion centre and a final decision is provided [5]. Sensing the final result depends on soft and hard combinations in soft combination bandwidth of spectrum is large and forward sensing result to fusion centre and in hard combination limited bandwidth is used and sensing data is quantized then sent to the fusion centre. In [6], the authors concentrate on hard combination scheme which has quantized sensing data, limited bandwidth and one-bit decision are used from local sensing for a final decision.

To improve the performance of cognitive radio artificial intelligence and machine learning techniques are used in the learning process [7]. In this paper, we mainly apply the fuzzy logic rule to get a final decision at the fusion centre and Fuzzy Logic Technique Primarily Energy and bandwidth considered as performance parameters. Fuzzy Membership Functions are calculated using energy at the first stage in local spectrum sensing Energy Detection method is used further in the second stage fuzzy logic technique is used [8]. Calculated membership functions are transferred to the fusion centre using the noiseless channel. The paper [9] explains Machine learning usage in Cooperative Spectrum Sensing using fuzzy techniques also gives an idea of fuzzy rules. The performance of detection is measured using the 
conventional method i.e. Energy detection method and using fuzzy logic machine learning technique [11]. For all methods Probability of Detection $\left(P_{d}\right)$, Probability of false alarm $\left(P_{f a}\right)$ and total error rate $\left(P_{e}\right)$ is calculated.

In this paper mainly, the final decision depends on more than one parameter like energy, number of samples and SNR value. Calculation of membership function depends on all three parameters. Proposed fuzzy logic uses energy values which are not detected by energy detection and give much accuracy also increases detection performance.

The remainder of this paper is organized as follows: The problem of Spectrum sensing and energy detection described in section 2. Overview of the system model is given in section 3. Our proposed cooperative spectrum sensing using fuzzy logic scheme is explained in section 4. Comparison with simulation results is described in section 5. Finally, the conclusion is given in section 6 .

\section{SPECTRUM SENSING MODEL}

Spectrum sensing is a most important process performed by cognitive radio. It allows the secondary user to sense the environment by detecting the transmission of the primary user so that it occupied frequency band.

Sensing process depends on two hypotheses

$$
\begin{gathered}
H_{0} \text { exist if } p(t)=c(t) \\
H_{1} \text { exist if } p(t)=g(t)+c(t)
\end{gathered}
$$

$p(t)$ is the received signal at a cognitive radio,

$g(t)$ is the transmitted signal from the primary user,

$c(t)$ is the noise signal at AWGN,

$H_{0}$ is the absence of primary user and

$H_{1}$ is the presence of primary user

Detector output or energy $(E)$ is compared to a threshold $(\lambda)$ to make sensing about the presence or absence of primary user. If $E>\lambda$, the $H_{1}$ hypothesis exist and if $E<\lambda, \mathrm{H}_{0}$ hypothesis exist.

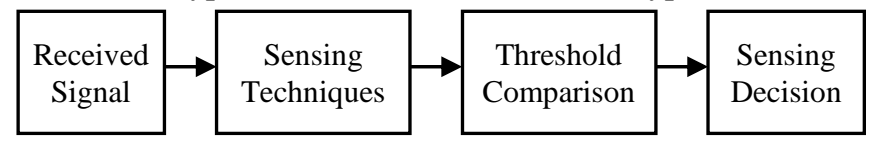

Fig.1. Spectrum sensing model

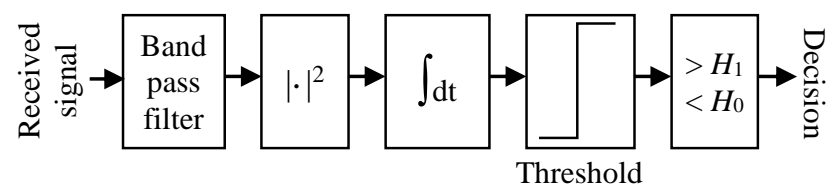

Fig.2. Energy detection technique

Mainly the energy detection method is used for spectrum sensing because it has long computation and execution complexity depending on information like frequency and bandwidth of the signal. For the energy detection, we can take an input signal which selects the bandwidth and it is sampled. Then it uses the implementation for FFT (Fast Fourier Transform), and then the absolute value sampled is squared. At last, we compared with between the output of the integrator and threshold. It is the presence or absence primary user can be detected [10].
Spectrum sensing does not require high processing time but error occur due to interference, shadowing, fading and hidden node problem so to overcome that we switch to cooperative spectrum sensing.

\section{COOPERATIVE SPECTRUM USING ENERGY DETECTION}

SENSING

Cooperative spectrum sensing occurs when a group of $\mathrm{CR}$ shares the sensing information received for PU detection. This provides a more accurate spectrum sensing on the specified given bandwidth area where the Secondary users present.

In centralized of cooperative spectrum sensing, there is a fusion centre (FC) within the network that collects the sensing information from all the sense CRs within the network. For data cooperative, all CRs are tuned to a control channel where a physical point-to-point link between each cooperating CR and the Fusion centre for sending the sensing results is called a reporting channel. Then fusion centre analyses the information and determines the bands that can and cannot be used.

In the fusion centre, mainly logical AND and logical OR rule is applied to the collected data. Depending on the final result which is calculated by rules presence or absence of the primary user in a given spectrum.

\section{SYSTEM MODEL}

Designing of the model is start with selecting the suitable RF environment. Cooperative spectrum sensing used to solve fading, shadowing, and hidden node problems. Single Primary user and $N$ number of cognitive radio users considered. Using energy detection method cognitive radio will detect if primary signal present or not and that decision is sent to the fusion centre through the control channel. Mainly energy parameter considered at each cognitive radio to sense the environment. The local decision of individual cognitive radio is used to find a final decision. At the fusion centre, the machine learning algorithm will be applied mainly fuzzy rules. Depending on that final decision will be taken at the fusion centre and analyze it.

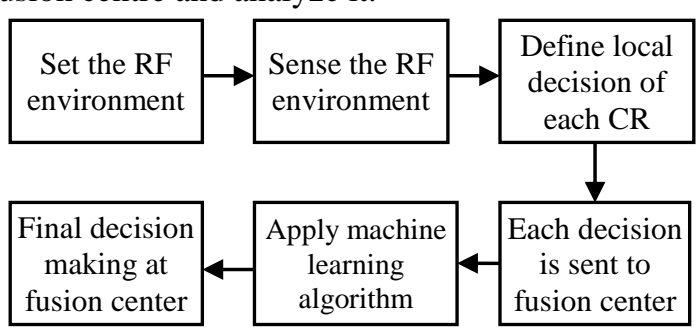

Fig.3. System model

\section{PROPOSED COOPERATIVE SPECTRUM SENSING SCHEME USING FUZZY LOGIC TECHNIQUE}

In a proposed system for detection of the primary user signal, the homogeneous network is considered. The network consists of a single primary user and 10 secondary users. Primary User transmit Amplitude Modulation signal, Transmitted signal pass through AWGN channel where noise is mixed with signal and it 
is received by cognitive radio as input. In Cooperative Spectrum Sensing more than one cognitive radio receive primary user signal. So, at each cognitive radio classification of signal and noise take place depending on the energy detection method. For the energy detection, we can take an input signal which selects the bandwidth and it is sampled. Then it uses the implementation for FFT (Fast Fourier Transform), and then the absolute value sampled is squared to calculate Energy Value of each cognitive radio. After calculating the energy of each cognitive radio, to calculate the total energy of Cooperative Spectrum Sensing system we multiple path loss to each cognitive radio's energy value and sum all cognitive radios Energy values to obtain total energy of the system. After that compare it against a predefined threshold value. If calculated Energy is greater than Threshold considering $H_{1}$ hypothesis which means in received signal primary user signal is present or if Energy is less than threshold only noise is present in the received signal.

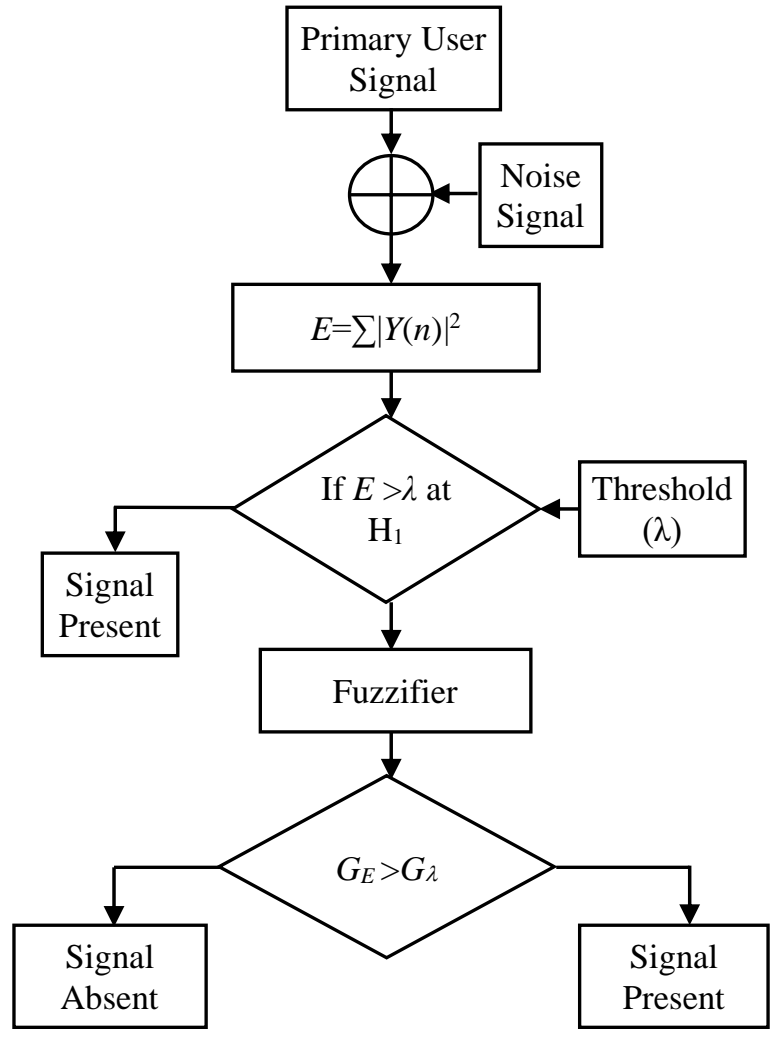

Fig.4. Flowchart of Proposed Scheme

Sometimes energy detection method will not detect the presence of Primary user properly, a confused state occurs where miss detection of signal take place so in that case, the secondary users will use a fuzzy logic scheme to decide the actual conditions [11]. In that Energy Values of H0 hypothesis used to calculate fuzzy membership functions. Fusion Threshold $\left(G_{\lambda}\right)$ is calculated using fuzzy false alarm rate values. Calculated fuzzy membership functions sent to the Fusion Center (FC) where different fuzzy fusion rules are applied like union rule, intersection rule, algebraic rule and algebraic product rule to get global membership function. [10] This Global membership function is compared with the fusion threshold. If global membership function is greater than the fusion threshold it means the primary user signal present otherwise primary signal is absent. Detection of the signal using fuzzy logic technique much better compare to the conventional method also fuzzy logic technique detect signal at low SNR condition.

For, Cooperative spectrum sensing, mainly narrow bandwidth is considered and depending on bandwidth Number of Samples are calculated here 200 number Samples are considered.

The proposed System consist of Two Phases:

i. Energy Detection Cooperative Spectrum Sensing

ii. Fuzzy Logic Cooperative Spectrum Sensing

We considered Primary user signal as Amplitude modulation signal which is passed through AWGN channel where along with transmitted signal noise is added and received by cognitive radio. In Cooperative Spectrum Sensing more than one CRs receive a primary user signal.

The energy of the Received signal by each cognitive radio is calculated as:

$$
E=\sum_{n=0}^{N}|y(n)|^{2}
$$

After calculating Energy of each cognitive radio to calculate the total energy of Cooperative Spectrum Sensing System Path Loss is multiplied with individual cognitive radio's energy and sum all cognitive radios Energy value to get total energy of the system.

$$
T E=\sum\left(E \times P_{L}\right)
$$

where, $T E$ is the total energy of cooperative spectrum sensing, $E$ is the Energy of individual CR and $P_{L}$ is the Path Loss

The threshold value is calculated as follows for the comparison.

$$
\lambda=\Gamma^{-1}\left(1-P_{f a}, N\right)
$$

where, $\lambda$ is the threshold, $P_{f a}$ is the Probability of false alarm rate, $N$ is the number of samples and $\Gamma^{-1}$ is the gamma inverse function

By comparing $T E$ and $\lambda$ value, the decision of the primary user signal is taken.

If $T E \geq \lambda$ then PU is present otherwise PU is absent.

If $(T E>\lambda)$, then $H_{1}$ hypothesis exist or

If $(T E<\lambda) H_{0}$ hypothesis exist.

Energy detection method will not detect the presence of primary user properly, a confused state occurs where miss detection of signal take place so in that case, the secondary users will use a fuzzy logic scheme to decide the actual conditions [11]. Machine Learning methods mainly adapt to learn about the surrounding environment. Energy Detection has SNR threshold below which it will fail to operate robustly but using fuzzy logic technique detection can be possible even at low SNR condition.

For Fuzzy Logic System, we need to calculate fuzzy membership functions, fusion threshold, and fuzzy false alarm rate. So, Energy values from $H_{0}$ hypothesis of Spectrum sensing used to create fuzzy membership functions and fuzzy false alarm rate. [10]

Fuzzy Probability of false alarm rate is calculated as follows

$$
\rho_{f}=Q\left(\frac{Z_{i}-N}{\sqrt{2 N}}\right)
$$


where, $Z_{i}$ is the energy value at $H_{0}$ hypothesis, $N$ is the number of samples, $\rho_{f}$ is the fuzzy false alarm rate and $Q$ is the $Q$ function

Using the probability of fuzzy false fusion threshold is calculated using inverse gamma function such as [12]

$$
\lambda_{F C}=\Gamma^{-1}\left(1-\rho_{f}, N\right)
$$

where, $\lambda_{F C}$ is the fusion threshold

After calculating the Fusion Threshold $\left(\lambda_{F C}\right)$ normalize each threshold values so that its range from 0 to 1 .

Fuzzy membership functions are calculated to apply fuzzy rules using them and calculate global membership function which is compared with the Fusion threshold for a final decision at the fusion centre.

Along with Energy and Number of Samples, SNR is considered to Calculate Fuzzy membership functions of the secondary user for applying fuzzy fusion rules at the fusion centre such as

$$
\rho_{d}=Q\left(\frac{Z_{i}-N(1+S N R)}{\sqrt{2 N(1+2(S N R))}}\right)
$$

where, $\rho_{d}$ is the fuzzy membership function, $Z_{i}$ is the energy value of $H_{0}$ hypothesis, $S N R$ is the SNR value at $\mathrm{dB}$ and $N$ is the number of samples

For calculation of global membership function, we apply fuzzy rules to fuzzy membership functions. Calculated fuzzy membership functions are sent to the fusion centre through reporting channel. Four hard combination fuzzy rules are applied to fuzzy membership functions. [10]

Intersection Rule:

$$
\rho_{F C}=\operatorname{Minimum}\left(\rho_{d 1}, \rho_{d 2}, \rho_{d 3}, \rho_{d 4}, \ldots, \rho_{d M}\right)
$$

where $M=100$. In Intersection Rule we calculate minimum value among the fuzzy membership function.

\section{Union Rule:}

$$
\rho_{F C}=\operatorname{Maximum}\left(\rho_{d 1}, \rho_{d 2}, \rho_{d 3}, \rho_{d 4}, \ldots, \rho_{d M}\right)
$$

where $M=100$. In Union Rule we calculate maximum value among the fuzzy membership function.

Algebraic product Rule:

$$
\rho_{F C}=\prod_{i=0}^{M} \rho_{d}
$$

where $M=100$, In Algebraic Product Rule, Product of fuzzy membership functions are calculated which gives global membership function.

Algebraic sum rule:

$$
\rho_{F C}=1-\prod_{i=0}^{M}\left(1-\rho_{d}\right)
$$

where $M=100, \rho_{F C}$ is the global membership function and $\rho_{d}$ is the fuzzy membership function.

In algebraic sum rule, product of fuzzy membership functions are calculated which gives global membership function.

After computing global membership function $\left(\rho_{F C}\right)$ by applying fuzzy rules for each rule's global membership function is compared with Fusion Threshold and the final decision is taken about the presence or absence of PU.

$$
\begin{aligned}
& \text { If } \rho_{F C}>\lambda_{F C}, \text { then the signal is present } \\
& \text { If } \rho_{F C}<\lambda_{F C} \text {, then the signal is absent }
\end{aligned}
$$

The proposed fuzzy logic System gives detection performance much better than compared to reference fuzzy logic system for all the fuzzy fusion rules. Also, for calculating fuzzy membership functions, it uses parameters like energy, a number of samples and SNR. Sensing of primary user signal is performed at low SNR condition. Here at $-10 \mathrm{~dB}$ signal detection is performed.

\section{SIMULATION RESULTS}

We presented the simulation results of all algorithms in details and we have analyzed the results. The results are obtained using MATLAB R2016a software. The cooperative spectrum sensing system is implemented using energy detection and fuzzy logic technique. The parameters for simulation is shown in Table.1.

Table.1. Parameters used for Simulation

\begin{tabular}{|c|c|}
\hline Simulation Parameters & Value \\
\hline Number of Samples & 200 \\
\hline Number of primary users & 1 \\
\hline Number of secondary users & 10 \\
\hline SNR values & $-10 \mathrm{~dB}$ \\
\hline
\end{tabular}

Using proposed fuzzy logic technique, we represented simulation results for $P_{d}$ vs. $P_{f a}, P_{m}$ vs. $P_{f a}$ graphs comparing all the fuzzy fusion rules of hard combinations such as union rule, intersection rule, algebraic sum rule, and algebraic product rule at -10dB SNR values using 200 numbers of samples. Results represent performance of detection much better compare to conventional energy detection method.

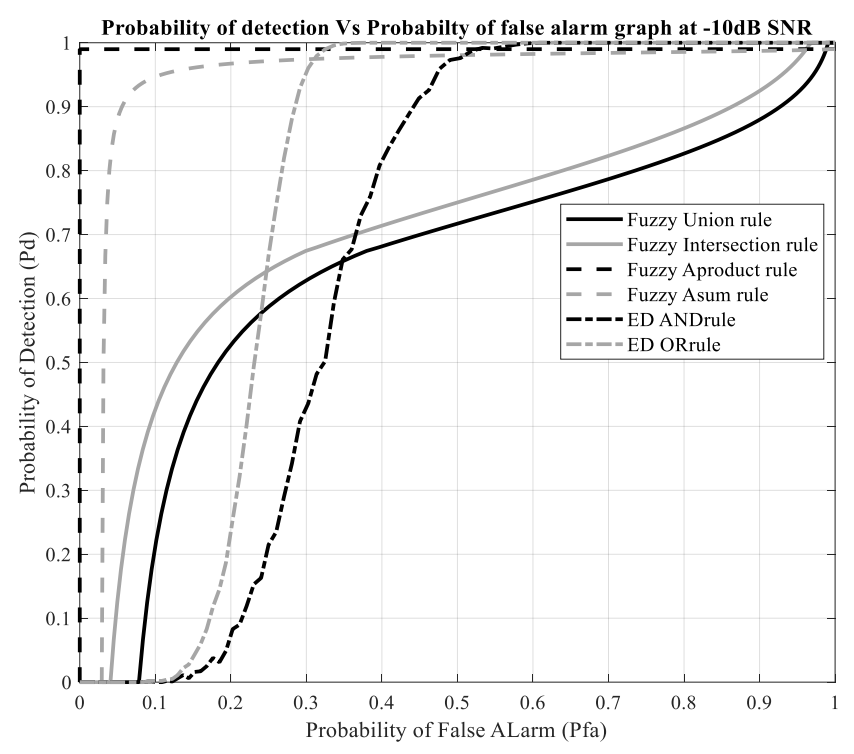

Fig.5. Comparison of ROC graph at $-10 \mathrm{~dB}$

The system performance is measured by considering $P_{d}, P_{f a}$, and parameters. Using ROC curve performance of the system is measured. ROC curve simulated under AWGN channel for logical AND rule and logical OR rule of energy detection and union rule, intersection rule, algebraic sum rule, and algebraic 
product rule of fuzzy logic techniques $-10 \mathrm{~dB}$ SNR of 10 Secondary Users using 200 numbers of samples is shown in Fig.5.

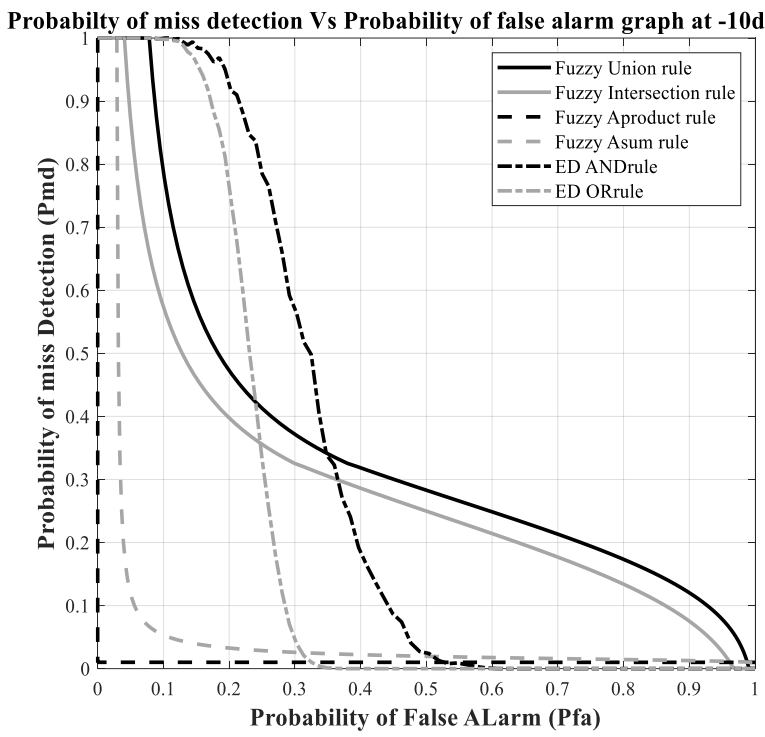

Fig.6. Comparison of $P_{m d}$ vs. $P_{f a}$ graph at $-10 \mathrm{~dB}$

The Fig.6 shows the results of $P_{m d}$ vs. $P_{m d}$ simulated under AWGN channel for logical AND rule and logical OR rule of energy detection and union rule, intersection rule, algebraic sum rule, and algebraic product rule of fuzzy logic techniques -10dB SNR of 10 Secondary Users using 200 number of samples.

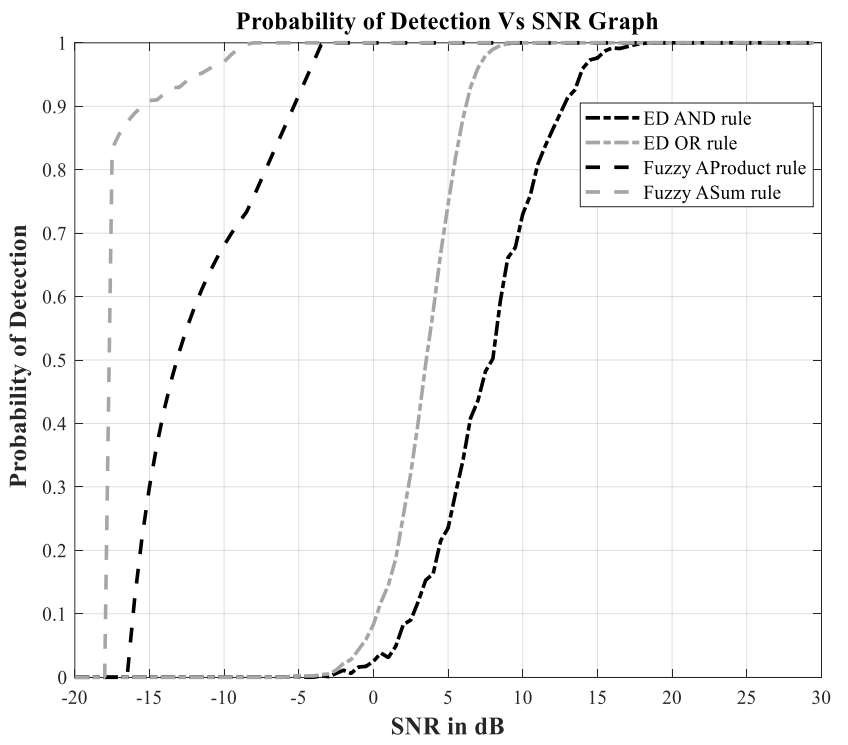

Fig.7. Comparison of $P_{d}$ vs. SNR graph

The graph represents a comparison of SNR vs Pd with a range of -20 to $30 \mathrm{~dB}$ of 10 secondary users for algebraic sum and algebraic product fuzzy fusion rules of fuzzy logic technique and compared with conventional logical OR and logical AND rules using 200 numbers of samples is shown in Fig.7.

The Fig. 8 represents a comparison of SNR vs. $P_{e}$ with a range of -20 to $30 \mathrm{~dB}$ of 10 secondary users for algebraic sum and algebraic product fuzzy fusion rules of fuzzy logic technique and compared with conventional logical OR and logical AND rules using 200 numbers of samples.

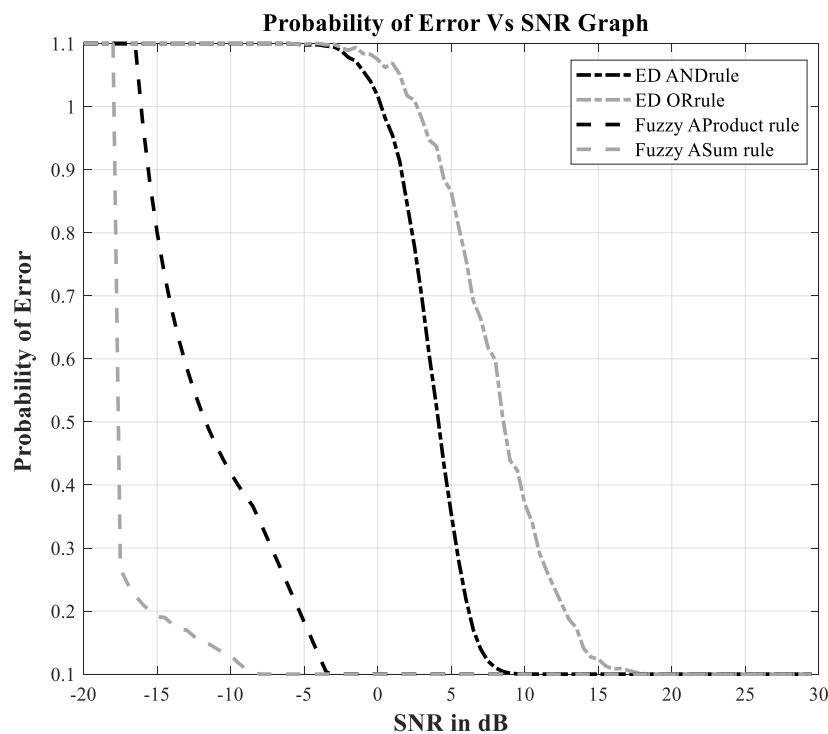

Fig.8. Comparison of $P_{e}$ vs. SNR graph

Table.2. Comparison of Different Cooperative Spectrum Sensing Techniques:

\begin{tabular}{|c|c|c|c|}
\hline Parameters & $\begin{array}{c}\text { Conventional } \\
\text { Energy } \\
\text { Detection } \\
\text { Cooperative } \\
\text { Spectrum } \\
\text { sensing }\end{array}$ & $\begin{array}{c}\text { Referred } \\
\text { Cooperative } \\
\text { Spectrum } \\
\text { Sensing } \\
\text { Scheme using } \\
\text { Fuzzy Logic } \\
\text { Technique [9] }\end{array}$ & $\begin{array}{c}\text { Proposed } \\
\text { Cooperative } \\
\text { Spectrum } \\
\text { Sensing } \\
\text { Fuzzy Logic } \\
\text { Technique }\end{array}$ \\
\hline $\boldsymbol{P}_{\boldsymbol{d}}$ at -10dB & 0.83 to 1 & 0.85 to 0.95 & 0.95 to 0.99 \\
\hline $\boldsymbol{P}_{\boldsymbol{e}}$ at -10dB & 0.21 & - & 0.1101 \\
\hline Training time & - & $2 \mathrm{~ms}$ & $2 \mathrm{~ms}$ \\
\hline $\begin{array}{c}\text { Implementation } \\
\text { Complexity }\end{array}$ & Less & High & High \\
\hline $\begin{array}{c}\text { Performance } \\
\text { Parameters }\end{array}$ & $\lambda$ & $Z_{i}$ & $Z_{i}$ and SNR \\
\hline
\end{tabular}

\section{CONCLUSIONS}

In this paper cooperative spectrum, sensing scheme using fuzzy logic technique is proposed. From results, it is inferred that the probability of false rate, the probability of detection and SNR plays a crucial role in deciding the performance of spectrum sensing. It can be enhanced by increasing the value of SNR or by decreasing the value of the probability of false rate. The Proposed Fuzzy Logic Technique achieves the highest performance in terms of $P_{e}, P_{d}$ and $P_{f a}$ compared to the other algorithms also it detects PU signal at low SNR condition compares to other algorithms. At $-10 \mathrm{~dB}$, the probability of error obtained is 0.1101 . The energy detection method is very easy to implement but it has poor performance at low SNR and its computational time is approximately 10s. But the Fuzzy Logic method is complex to implement but it gives much better performance at low SNR condition and gives $98 \%$ of detection performance. 


\section{REFERENCES}

[1] N. Muchandi and R. Khanai, "Cognitive Radio Spectrum Sensing: A Survey", Proceedings of International Conference on Electrical, Electronics, and Optimization Techniques, pp. 3233-3237, 2016.

[2] M.Z. Alom, T.K. Godder and M.N. Morshed, "A Survey of Spectrum Sensing Techniques in Cognitive Radio Network", Proceedings of International Conference on Advances in Electrical Engineering, pp. 161-164, 2015.

[3] M. Bkassiny, Y. Li and S. Jayaweera, "A Survey on Machine-Learning Techniques in Cognitive Radios", IEEE Communications Surveys and Tutorials, Vol. 15, No. 3, pp. 1136-1159, 2013.

[4] N. Swetha, P.N. Sastry and Y.R. Rao, "Analysis of Spectrum Sensing Based on Energy Detection Method in Cognitive Radio Networks", Proceedings of International Conference on IT Convergence and Security, pp. 1-4, 2014.

[5] I. Akyildiz, W. Lee, M. Vuran and S. Mohanty, "Next Generation/Dynamic Spectrum Access/Cognitive Radio Wireless Networks: A Survey", Computer Networks, Vol. 50, No. 13, pp. 2127-2159, 2006.

[6] N. Zhao, F. Yu, H. Sun and A. Nallanathan, "A EnergyEfficient Cooperative Spectrum Sensing Schemes for Cognitive Radio Networks", EURASIP Journal on Wireless Communications and Networking, Vol. 6, No. 1, pp. 1-7, 2013.

[7] Kuldeep Kaur and Inderdeep Kaur Aulakh, "Optimization of Cooperative Spectrum Sensing using Fuzzy Logic",
International Journal of Application or Innovation in Engineering and Management, Vol. 3, No. 11, pp. 1-8, 2014.

[8] N. Abbas, Y. Nasser and K. Ahmad, "Recent Advances in Artificial Intelligence and Learning Techniques in Cognitive Radio Networks", EURASIP Journal on Wireless Communications and Networking, Vol. 8, No. 1, pp. 23-28, 2015.

[9] A. Mohammadi and Taban, "Cooperative Spectrum Sensing using Fuzzy Membership Function of Energy Statistics", AEU-International Journal of Electronics and Communications, Vol. 70, No. 3, pp. 234-240, 2016.

[10] C. Satrio and J. Jaeshin, "Two-Stage Spectrum Sensing Scheme using Fuzzy Logic for Cognitive Radio Networks", Journal of Information and Communication Convergence Engineering, Vol. 14, No. 1, pp. 1-8, 2016.

[11] N. Arora and R. Mahajan, "Cooperative Spectrum Sensing using Hard Decision Fusion Scheme", International Journal of Engineering Research and General Science, Vol. 2, No. 4, pp. 1-8, 2014.

[12] T. Kieu-Xuan, "A Cooperative Spectrum Sensing Scheme using Fuzzy Logic for Cognitive Radio Networks", KSII Transactions on Internet and Information Systems, Vol. 4, No. 3, pp. 289-304, 2010.

[13] D. Teguig, B. Scheers and V. Le Nir, "Data Fusion Schemes for Cooperative Spectrum Sensing in Cognitive Radio Networks", Proceedings of IEEE International Conference on Military Communications and Information Systems, pp. 881-887, 2012. 